\title{
Harmonizing Standards and Incentives in Medical Device Regulation: Lessons Learned From the Parallel Review Pathway
}

\section{Citation}

Holtzman, Jessica N. 2019. Harmonizing Standards and Incentives in Medical Device Regulation: Lessons Learned From the Parallel Review Pathway. Doctoral dissertation, Harvard Medical School.

\section{Permanent link}

http://nrs.harvard.edu/urn-3:HUL.InstRepos:41971472

\section{Terms of Use}

This article was downloaded from Harvard University's DASH repository, and is made available under the terms and conditions applicable to Other Posted Material, as set forth at http:// nrs.harvard.edu/urn-3:HUL.InstRepos:dash.current.terms-of-use\#LAA

\section{Share Your Story}

The Harvard community has made this article openly available. Please share how this access benefits you. Submit a story. 
Scholarly Report submitted in partial fulfillment of the MD Degree at Harvard Medical School

Date: 25 January 2019

Student Name: Jessica Holtzman, B.A.

Scholarly Report Title: Harmonizing Standards and Incentives in Medical Device Regulation: Lessons Learned from the Parallel Review Pathway

Mentor Name(s) and Affiliations: Daniel B. Kramer, MD, MPH, Assistant Professor of Medicine, Richard and Susan Smith Center for Outcomes Research in Cardiology, Beth Israel Deaconess Medical Center 


\section{TITLE - Harmonizing Standards and Incentives in Medical Device Regulation: Lessons Learned from the Parallel Review Pathway}

Background: The US Food and Drug and Administration (FDA) and Centers for Medicare and Medicaid Services (CMS) both play key roles in evaluating new medical devices. In 2011, they jointly launched the Parallel Review Pathway as a means for manufacturers of high-risk medical devices to meet the review requirements of FDA and CMS simultaneously. Through this model, CMS undertakes evaluation for a national coverage determination in conjunction with the submission of clinical trial and other data to the FDA for its review, rather than the traditional timing after FDA approval. The purpose of this article is to review the initial promise of the parallel review pathway and consider explanations for its unmet expectations.

Methods: We review the underlying support for parallel review, characterize its outcomes to date, and consider reasons why its utilization has not met expectations. We highlight examples of supporting evidence used to We propose solutions for increasing participation in parallel review as a means towards increasing regulatory efficiency alongside strengthening evidence of safety and effectiveness among Medicare beneficiaries.

Results: Despite a strong theoretical grounding, only 3 devices have completed parallel review, with a $4^{\text {th }}$ under evaluation. Two devices received joint approval from CMS and FDA, while one device was rejected by the FDA for lack of clinical evidence demonstrating efficacy when compared to control.

Conclusions: This analysis reveals shortcomings of parallel FDA and CMS review for medical devices and provides important lessons for understanding manufacturers' incentives, risk tolerance, and the remaining barriers for streamlining device review while preserving high standards that protect public health. 


\section{Table of Contents}

Page 1 - Title Page

Page 2 - Abstract

Page 3 - Table of Contents, Citation, Link to Article, Glossary of Abbreviations

Page 4 - Summary of Scholarly Question and Contribution to Work

Page 5 - Appendix I: Published manuscript

Citation: Holtzman JN \& Kramer DB. The Journal of Law, Medicine, and Ethics, 46 (2018): 1034-1039.

\section{$\operatorname{Link}(s)$ :}

$\underline{\text { https://journals.sagepub.com/doi/full/10.1177/1073110518822005 }}$

https://doi.org/10.1177/1073110518822005

\section{Glossary of abbreviations}

FDA - Food and Drug Administration

CMS - Centers for Medicare and Medicaid Services

NCD - National Coverage Determination

CDRH - Center for Devices and Radiologic Health

S-ICD - Subcutaneous Implantable Cardioverter Defibrillator

MADIT S-ICD - Multicenter Automatic Defibrillator Implantation Trial with Subcutaneous Implantable Cardioverter Defibrillator 
In 2011, the US Food and Drug and Administration (FDA) and Centers for Medicare and Medicaid Services (CMS) launched the Parallel Review Pathway as a means for manufacturers of high-risk medical devices to meet the review requirements of FDA and CMS simultaneously. Through this model, CMS undertakes evaluation for a national coverage determination in conjunction with the submission of clinical trial and other data to the FDA for its review, rather than the traditional timing after FDA approval. Despite a strong theoretical grounding, only 3 devices have completed parallel review, with a $4^{\text {th }}$ under evaluation.

Through this scholarly project, we sought to review the underlying support for the Parallel Review pathway, characterize its outcomes to date, and consider reasons why its utilization has not met expectations. We sought to propose solutions for increasing participation in parallel review as a means towards increasing regulatory efficiency alongside strengthening evidence of safety and effectiveness among Medicare beneficiaries.

This project originally arose out of a desire to better characterize the landscape of medical device regulatory policy, tying together ethics, policy, and clinical outcomes, specifically as they relate to cardiac and other medical devices. Pharmaceutical regulatory policy is relatively better understood, but little effort has been put toward delineating strengths and challenges of the complex medical device regulatory landscape. The first stage of this project involved researching the historical landscape of medical device regulation within the United States and the various routes to market for medical devices. Using several legal textbooks, as well as primary guidance documents from the Food and Drug Administration and Centers for Medicare and Medicaid Services, I constructed a comprehensive document covering medical device policies including the Pre-Market Approval, PreMarket Approval Supplement, Humanitarian Device Exemption, Product Development Protocol, Reclassification, 510(k), De Novo Classification, Investigational Device Exemption, and Custom Device Exemption. Additionally, the report characterized the Parallel Review Pathway, the Third Party Review pathway, and the Breakthrough Device Program. We also addressed the funding for such programs including the Prescription Drug User Fee Act and the Medical Device User Fee Act.

From that point, we honed in more specifically on the Parallel Review pathway, identifying four devices that have undergone the pathway, analyzing their regulatory outcomes, and reaching out to subject matter experts at the Food and Drug Administration for further comment on the pathway. I constructed the original draft of the manuscript, with significant content and subject matter guidance and contributions, as well as editing, by Dr. Kramer. 


\section{Harmonizing Standards and Incentives in Medical Device Regulation: Lessons Learned from the Parallel Review Pathway}

\section{Health Policy Portal}

\author{
Jessica N. Holtzman and \\ Daniel B. Kramer
}

\section{About This Column}

Aaron S. Kesselheim serves as the editor for Health Policy Portal. Dr. Kesselheim is the JLME editor-inchief and director of the Program On Regulation, Therapeutics, And Law at Brigham and Women's Hospital/Harvard Medical School. This column features timely analyses and perspectives on issues at the intersection of medicine, law, and health policy that are directly relevant to patient care. If you would like to submit to this section of JLME, please contact Dr. Kesselheim at akesselheim@bwh.harvard.edu.
In late 2017, the US Food and Drug Administration (FDA) and Centers for Medicare and Medicaid Services (CMS) jointly approved Foundation Medicine's FoundationOne CDx, a comprehensive diagnostic and genomic profiling assay for solid tumors that is intended to inform the use of targeted oncology therapies. The decision was only the second affirmative determination to be reached via the Parallel Review Pathway, which was launched in 2011 as a means for manufacturers of high-risk medical devices to meet the review requirements of FDA and CMS simultaneously. Under this review process, CMS assesses its national coverage determination concurrently with review of safety and effectiveness data by the FDA. This pathway was designed to build on the agencies' overlapping mandates and "common interest in improving the health of patients through the availability of safe, effective, and affordable medical products,"1 while enhancing the speed and transparency of decision-making that leads to the sale of new medical devices. But why, if some policymakers and the medical device industry suggest that the US market needs more regulatory efficiency, has parallel review been used so infrequently? We sought to review the initial promise of the parallel review pathway and consider explanations for its unmet expectations.

Approval and Coverage of Medical Devices in the US

In the US, new high-risk medical devices must undergo formal FDA review before introduction to the market. ${ }^{2}$ Manufacturers of devices such as coronary stents and implantable cardioverter-defibrillators, intended for life-sustaining or lifesupporting purposes, most often submit applications via the Premarket Approval (PMA) process. While specific requirements are tailored to device type, the PMA pathway involves developing preclinical and clinical data that demonstrate "reasonable assurance of safety and effectiveness."3 This standard involves sufficient valid scientific evidence to demonstrate that the probable health benefits attributable to the intended use of the device outweigh any probable risks, and that such benefits result in clinically meaningful results. ${ }^{4}$ The FDA has historically considered "valid scientific evidence" for the determination of effectiveness to consist of well-controlled clinical investigations, although many highrisk devices are approved based on single-arm trials without active comparators or other less rigorous data sources, with evidentiary standards tailored to the device and clinical context. ${ }^{5}$

Once FDA approval is granted, a device sponsor must submit an application to CMS to determine whether the product is "reasonable and necessary," which are Medicare's statutory coverage criteria. Necessity has been broadly interpreted to reflect the strength and rigor of the clinical evidence supporting a device, taking into account factors including study design and sample size. ${ }^{6}$ By contrast, the meaning of "reasonable" has not

Jessica N. Holtzman, B.A., holds a B.A. in Human Biology from Stanford University (Palo Alto, CA), is a medical student at Harvard Medical School (Boston, MA), and a research student at the Richard A. and Susan F. Smith Center for Outcomes Research in Cardiology at BIDMC. Daniel B. Kramer, M.D., M.P.H., earned his A. B. in Philosophy from Brown University (Providence, RI), M.D. from Harvard Medical School, and M.P.H. from the Harvard TH Chan School of Public Health (both in Boston, MA). He is a cardiac electrophysiologist at BIDMC, where he is also core faculty at the Richard A. and Susan F. Smith Center for Outcomes Research in Cardiology. 
been clearly defined with regard to medical devices. Some have postulated that this refers to devices that have sufficient evidence to support clinically-relevant improved health outcomes, ${ }^{7}$ achieved through assessment of clinical data for study quality, relevance to Medicare beneficiaries, and magnitude of benefits and risks (Table 1). ${ }^{8}$

Responsibility for making the reasonable and necessary determination for the vast majority of devices falls to fiscal intermediaries that serve as representatives of different Medicare districts within the US. These contractors assess whether a device meets the reasonable and necessary criteria, resulting in a local coverage determination. Local coverage decisions may limit the coverage of items or services to specific diagnoses, or may preclude coverage entirely, with each decision applicable within the contractor's local jurisdiction. ${ }^{9}$

Alternatively, manufacturers, clinicians, or CMS itself may request a national coverage determination (NCD), which grants, limits, or excludes Medicare coverage nationwide, particularly when significant uncertainty regarding the risks and benefits of a new device exists or when non-uniform local coverage determinations exist. NCDs are issued on approximately 5-15 devices or services annually, and are targeted at those posing special concerns related to cost, safety, or effectiveness among Medicare beneficiaries. Until 2017, NCDs were published prior to implementation and local coverage decisions were not publicly available. However, under the 21st Century Cures Act, fiscal intermediaries were also required to publish the full local coverage decision, public comments and responses, a summary of the evidence considered, and the rationale for the determination, thereby clarifying the data upon which these determinations are decided. ${ }^{10}$

In practice, most applications for NCDs are approved. Among NCDs made by CMS from 1999-2007, a favorable decision was reached in more than $60 \%$ of the cases. ${ }^{11}$ Another potential outcome could be issuance by CMS of "coverage with

Table I

Evidence Reviewed to Determine a Medical Device "Reasonable and Necessary" for National Coverage Determination as Delineated in CMS Decision Memos

\section{Device or Clinical Test \\ Oncologic Indications of FDG-PET ${ }^{35}$}

Decision: Coverage for use to guide subsequent management of antitumor treatment strategy after completion of initial anticancer therapy

\section{Evidence}

- External technology assessment - two large external summative reports (2010 Special Report from the Blue Cross Blue Shield Technology Evaluation Center on PET for posttreatment surveillance of cancer \& Report from United Kingdom entitled "Overview of the clinical effectiveness of positron emission tomography imaging in selected cancers")

- Internal technology assessment — articles submitted by requesters, cited in public comment, and derived from literature search of PubMed database restricted to high quality studies (2 prospective controlled trials, I I prospective cohort trials, 14 prospective case series, 17 retrospective case series, I case series or case report)

- Review of professional society position statements and evidence-based guidelines

Transcatheter Aortic Valve Replacement for Symptomatic Aortic Stenosis $^{36}$

Decision: Covered for the treatment of symptomatic aortic valve stenosis when pre-operative evaluation meets certain standards, Coverage with Evidence Determination for non-FDA approved indications

- External technology assessment - Belgian Health Technology Assessment, Interventional procedure overviews/guidance - NICE, Health technology assessment from California Technology Assessment Forum, and PARTNER study

- Internal technology assessment - Derived from literature search of PubMed database restricted to high quality studies (5 randomized controlled trials, I study addressing quality of life, I observational study of long-term outcomes, I study of complications, 3 studies of frailty)

- Primary focus on whether pivotal PARTNER randomized controlled trial was adequate to draw conclusions about health outcomes

- Review of ACCFIAATS/SCAI/STS Expert Consensus Guidelines

FDG-PET: fluorodeoxyglucose positron emission tomography; FDA: Food and Drug Administration; NICE: National Institute for Health and Care Excellence; PARTNER: Placement of Aortic Transcatheter Valves trial; ACCF: American College of Cardiology Foundation; AATS: American Association for Thoracic Surgery; SCAl: The Society for Cardiovascular Angiography and Interventions; STS: Society of Thoracic Surgeons.

evidence development," in which reimbursement for the device is provided only in the context of the collection of additional clinical data, such as through registries or clinical investigations. ${ }^{12}$ Approximately two dozen devices or services are currently subject to coverage with evidence development decisions stretching back to 2006. ${ }^{13}$

The existence of separate formal review processes for high-risk medi- 
cal devices at FDA and CMS has contributed to worries about the dissemination of new products and the possibility of delays in timely delivery of devices to market. ${ }^{14}$ The independent CMS process may introduce delays between FDA approval and CMS reimbursement for the medical device's utilization..$^{15}$ An analysis of the six NCDs issued by CMS in 2013 found that the average time from initiation of analysis to release of the proposed decision was approximately 6 months, with an additional 3 months from proposed to final decisions, and another 4 from posting of the final decision to publishing of implementation instructions. ${ }^{16}$ For example, the WATCHMAN device for left atrial appendage exclusion was FDA-approved in March of 2015, but only received an NCD from CMS in April of 2016. Though comparable data are not available regarding implementation of local coverage determinations, their timeline is generally thought to be more expeditious than that of national coverage. ${ }^{17}$

\section{The Parallel Review Process and its Underuse}

In June of 2010, the FDA and CMS signed a memorandum of understanding to work together "to promote initiatives related to the review and use of FDA-regulated drugs, biologics, medical devices, and foods" with the express purpose to "promote collaboration and enhance knowledge and efficiency by providing for the sharing of information and expertise between the Federal partners." ${ }^{18}$ By 2011, the parallel review pilot program was launched between the FDA's Center for Devices and Radiological Health (CDRH) and CMS. The program was designed not to alter the review criteria for either agency, but to shorten the review process and promote transparency and efficiency. In particular, earlier CMS involvement was thought to encourage clinical trial design that would promote data collection to serve the evidentiary requirements of the FDA and CMS, with a particular focus on therapeutics likely to impact Medicare beneficiaries.

To meet appropriateness criteria for consideration of participation in the parallel review pathway, the device manufacturer must (1) agree to meet jointly with FDA and CMS prior to initiating clinical investigation to incorporate feedback from both parties regarding trial design and analysis, (2) document that the device requires an application for premarket approval (or a petition for de novo review, which classifies a novel device that would otherwise be high-risk to a lower risk tier), and (3) demonstrate that the device is not statutorily excluded from Medicare's

Table 2

Medical Devices Evaluated via the Parallel Review Pathway

\begin{tabular}{|c|c|c|c|}
\hline Manufacturer, Device & $\begin{array}{l}\text { Date } \\
\text { of Joint } \\
\text { Approval }\end{array}$ & Purpose & Outcome \\
\hline $\begin{array}{l}\text { Exact Sciences, } \\
\text { Cologuard }\end{array}$ & $08 / 11 / 2014$ & $\begin{array}{l}\text { Qualitative detection of colorectal } \\
\text { neoplasia associated DNA markers and } \\
\text { presence of occult hemoglobin in stool }\end{array}$ & $\begin{array}{l}\text { Data in application support reasonable } \\
\text { assurance of safety and effectiveness of } \\
\text { device when used in accordance with } \\
\text { indications for use }\end{array}$ \\
\hline $\begin{array}{l}\text { Medtronic, Symplicity } \\
\text { Renal Denervation System }\end{array}$ & $\begin{array}{l}\text { Not } \\
\text { Approved }\end{array}$ & $\begin{array}{l}\text { Treatment of resistant hypertension } \\
\text { through renal-artery denervation }\end{array}$ & $\begin{array}{l}\text { Rejected by FDA. Phase III blinded trial } \\
\text { did not show significant decrease in } \\
\text { systolic pressure } 6 \text { months after renal- } \\
\text { artery denervation as compared with } \\
\text { sham control }\end{array}$ \\
\hline $\begin{array}{l}\text { Foundation Medicine, } \\
\text { FoundationOne CDx }\end{array}$ & $11 / 30 / 2017$ & $\begin{array}{l}\text { Oncologic assay to detect genomic } \\
\text { alterations in } 324 \text { genes using DNA } \\
\text { isolated from formalin-fixed tumor } \\
\text { tissue specimens using advanced } \\
\text { sequencing techniques }\end{array}$ & $\begin{array}{l}\text { Data in application support reasonable } \\
\text { assurance of safety and effectiveness of } \\
\text { device when used in accordance with } \\
\text { indications for use }\end{array}$ \\
\hline $\begin{array}{l}\text { Boston Scientific, } \\
\text { EMBLEM MRI S-ICD } \\
\text { System }\end{array}$ & $\begin{array}{l}\text { Decision } \\
\text { Pending }\end{array}$ & $\begin{array}{l}\text { Subcutaneous implantable cardioverter } \\
\text { defibrillator without transvenous pacing } \\
\text { leads }\end{array}$ & $\begin{array}{l}\text { MADIT S-ICD trial currently being } \\
\text { conducted to assess survival benefit } \\
\text { of ICD implantation in patients over } \\
65 \text { with history of prior heart attack, } \\
\text { diabetes, and moderately reduced } \\
\text { ejection fraction versus medical therapy }\end{array}$ \\
\hline
\end{tabular}

S-ICD: Subcutaneous Implantable Cardioverter Defibrillator; MADIT S-ICD: Multicenter Automatic Defibrillator Implantation Trial with Subcutaneous Implantable Cardioverter Defibrillator 
hospital and general medical benefit categories. ${ }^{19}$

By 2016, the parallel review process received 60 inquiries and 26 applications, with the program purporting to possess the resources to undertake review of 3-5 devices per year. However, since the program's announcement in 2011, only four manufacturers have publicly announced their participation in the dual-review process, with two affirmative, one negative, and one pending determination (Table 2). Reasons why not all 26 devices have been accepted by FDA and CMS for parallel review include existence of a prior NCD or ineligibility for an NCD determination. However, the details of applications and reasons for denial are not publicly available.

Several factors may explain why parallel review has received limited interest. First, the lack of utilization of the parallel review process likely reflects manufacturers' unwillingness to undertake the risk associated with seeking an NCD. ${ }^{20}$ In practice, most FDA-approved devices are covered through local coverage decisions. Thus, the benefits of expedited progression through FDA and CMS review may not be worth the risks of a negative determination that precludes or markedly limits reimbursement nationally for Medicare patients. ${ }^{21}$ Similarly, increased scrutiny from CMS may lead to additional post-market requirements (such as a mandatory registry), which may not be mandated by local administrative contractors, adding another layer of potential financial risk for sponsors. That said, it is unlikely that the fear of a restrictive NCD accounts entirely for low participation in the parallel pathway, as most devices that do go through the NCD process end up favorably reviewed.

Second, manufacturers often do not design device trials that demonstrate effectiveness in populations specifically relevant to CMS, which will necessarily include older patients who are more likely to have multiple comorbid conditions..$^{22}$ For example, a study of 82 studies published in 2015 supporting the approval of 42 high risk medical devices revealed that only $9 \%$ of trials analyzed effectiveness by age and that results by age were reported on only $7 \%$ of device labels. ${ }^{23}$ From a clinical and policy perspective, increasing testing of new products in such patients is an important public health goal because many of these patients will end up receiving approved high-risk devices. ${ }^{24}$ Yet evaluating safety and effective- ers early in the investigative process, had made it clear that high-quality evidence specific to older patients would be necessary for coverage of cardiac resynchronization therapy, clinical trials might have been redesigned accordingly. Ideally, parallel review would encourage exactly these study refinements rather than a calculated assumption that a new device

\section{Despite the strong rationale for parallel review, this pathway has not been used widely. Strengthening the incentives for sponsors to pursue parallel review may require new policy approaches that either increase the eligible device submissions, or shift the burden of proof regarding clinical effectiveness in Medicare beneficiaries more explicitly towards manufacturers.}

ness endpoints in this population may complicate outcomes ascertainment and increase safety concerns about devices since such patients are likely to be at greater risk for adverse events. $^{25}$

From a device manufacturer's standpoint, it is more advantageous - and less expensive - to demonstrate clinical improvements in a healthier and more homogenous younger population, and if necessary argue afterward for extrapolation to Medicare beneficiaries. For example, Medicare currently covers cardiac resynchronization therapy for patients with systolic heart failure and clinical characteristics aligned with pivotal clinical trials, despite its own technology assessment acknowledging the lack of trials focused on Medicare-eligible patients and older $(>75)$ patients in particular. ${ }^{26}$ But this permissive approach may not serve either patients' or CMS' interests well, as it is not at all clear that cardiac resynchronization therapy (particularly when paired with defibrillator back-up) actually improves outcomes meaningfully for older patients. ${ }^{27}$ If CMS, working with manufactur- or labeling will not be met with sufficient skepticism to merit a restrictive NCD.

\section{Future Directions}

Despite the strong rationale for parallel review, this pathway has not been used widely. Strengthening the incentives for sponsors to pursue parallel review may require new policy approaches that either increase the eligible device submissions, or shift the burden of proof regarding clinical effectiveness in Medicare beneficiaries more explicitly towards manufacturers.

Increasing eligibility for the program may be simpler. While initially conceived to focus on highrisk devices subject to PMA review, parallel review could be expanded to include selected medium-risk devices. Most medium-risk devices are evaluated through the 510k pathway, through which devices are evaluated based on whether they introduce new safety and effectiveness concerns compared to a predicate device already on the market to which they are compared. ${ }^{28}$ Notably, the $510 \mathrm{k}$ pathway has been criticized for inad- 
equately evaluating new devices and has even been recommended for replacement by a National Academy of Medicine panel, in part because most 510k applications do not include clinical data. ${ }^{29}$ At the same time, discordance between FDA and CMS decisions may be more common for $510 k$-cleared devices, ${ }^{30}$ suggesting an opportunity for sponsors to incorporate joint feedback from FDA and CMS in application design. Pairing requirements to include more routine collection of clinical data prior to marketing select mediumrisk devices with making these submissions eligible for parallel review might partially address criticism of the 510k pathway while also increasing program utilization. For example, it has been suggested that mediumrisk device types such as molecular diagnostics may be particularly well-suited to parallel review because of comparatively slow clearance. ${ }^{31}$ Newer technology subject to $510 \mathrm{k}$ clearance, such as selected mobile applications, might also be targeted for inclusion in parallel review as a means towards encouraging CMS to provide payment for services - such as review of arrhythmia recordings sent directly from patients to their providers - that currently are not eligible for reimbursement.

Alternatively, CMS might motivate program participation by adopting a more aggressive stance regarding requirements for data specific to Medicare beneficiaries. These patients have been shown to differ substantially in terms of age, gender, and comorbidities from study populations represented in cardiovascular device trials, ${ }^{32}$ while the impact of therapeutics on patients with multiple chronic conditions in particular remains an important public health concern. ${ }^{33}$ As the rigor of the local coverage decisions review has been called into question, ${ }^{34}$ it may be worthwhile for CMS to lower its threshold for considering NCDs for high-risk devices, and to specifically focus on the need for evidence generation among populations similar to Medicare beneficiaries prior to providing coverage. CMS could then require device manufacturers to col- lect clinical data specifically pertaining to Medicare beneficiaries prior to issuing approval for reimbursement. Such a strategy would require funding to allow CMS to afford greater resources to conduct a larger number of NCDs. This strategy would alter the assumption that new high-risk devices could earn reimbursement through local coverage decisions alone. If more sponsors anticipate that their products will be subject to NCDs, this would likely incentivize their simultaneous engagement with FDA and CMS through parallel review. Conversely, if sponsors continue to assume that the risks of attracting scrutiny through an NCD remain small, they will have little to gain from encouraging additional oversight or proactively testing their devices in older, sicker patients.

The infrequent use of parallel review to date suggests that device sponsors' criticism of the successive FDA and CMS review processes and pleas for efficiency reflect a broader desire for minimizing regulatory risk. However, this program holds the potential to buttress regulatory standards of both agencies, requiring increased data collection for older patients not often included in clinical trials, while making potentially lifesaving products available to the public more quickly. While time spent under review represents one element of risk to device manufacturers, sponsors also weigh potential restrictions on labeled indications and populations and post-market requirements when evaluating paths to market. These factors must be considered when motivating participation in parallel review, and may help the program achieve its goal of bringing useful medical devices to market, while also generating high-quality evidence specific to Medicare beneficiaries.

\section{Note}

Dr. Kramer is supported by the Greenwall Faculty Scholars Program in Bioethics, and is a consultant to the Circulatory Systems Advisory Panel of the Food and Drug Administration.

\section{References}

1. Centers for Medicare and Medicaid Services \& Food and Drug Admin- istration. Parallel review of medical products, available at <https:// www.federalregister.gov/documents/ 2010/09/17/2010-23252/parallelreview-of-medical-products > (last accessed November 27, 2018.

2. D.B. Kramer, S. Xu, and A.S. Kesselheim, "Regulation of Medical Devices in the United States and European Union," New England Journal of Medicine 366, no. 9 (2012): 848-855.

3. US Food and Drug Administration, Premarket Application Review Process, available at <https://www.fda.gov/ MedicalDevices/DeviceRegulationandGuidance/HowtoMarketYourDevice/ PremarketSubmissions/PremarketApprovalPMA/ucm047991.htm> (last accessed November 27, 2018).

4. U.S. FDA. Code of Federal Regulations. Title 21, Volume 8, Chapter I, Subchapter H, Part 860, available at <https://www.accessdata.fda.gov/ scripts/cdrh/cfdocs/cfCFR/CFRSearch. $\mathrm{cfm} ? \mathrm{FR}=860.7>$ (last visited November 27,2018 )

5. S.S. Dhruva, L.A. Bero, and R.F. Redberg, "Strength of Study Evidence Examined by the FDA in Premarket Approval of Cardiovascular Devices," JAMA 302, no. 24 (2009): 2679-2685; V.K. Rathi, H.M. Krumholz, and F.A. Masoudi, J.S. Ross, "Characteristics of Clinical Studies Conducted Over the Total Product Life Cycle of High-Risk Therapeutic Medical Devices Receiving FDA Premarket Approval in 2010 and 2011," JAMA 314, no. 6 (2015):604-612.

6. J.D. Chambers, K.E. May, and P.J. Neumann, "Medicare Covers the Majority of FDA-Approved Devices and Part B Drugs, but Restrictions and Discrepancies Remain," Health Affairs (Millwood) 32, no. 6 (2013):1109-1115.

7. S. Tunis, R. Berenson, S. Phurrough, and P. Mohr, "Improving the Quality and Efficiency of the Medicare Program through Coverage Policy Issues," Urban Institute (2011), available at <http://www.urban.org/sites/default/ files/alfresco/publication-pdfs/412392Improving-the-Quality-and-Efficiency-of-the-Medicare-Program-ThroughCoverage-Policy.PDF> (last accessed November 27, 2018); P.J. Neumann and J.D. Chambers, "Medicare's Enduring Struggle to Define "Reasonable and Necessary' Care, New England Journal of Medicine 367, no. 19 (2012): 1775-1777.

8. Centers for Medicare and Medicaid Services. Decision Memo for Positron Emission Tomography (FDG) for Solid Tumors (CAG-00181R4), available at $<$ https://www.cms.gov/medicare-coverage-database/details/nca-decisionmemo.aspx? NCAId $=263>$ (last accessed November 27, 2018)

9. Department of Health and Human Services, "Local Coverage Determinations Create Inconsistency in Medicare Coverage," available at <https://oig. 
hhs.gov/oei/reports/oei-01-11-00500. pdf $>$ (last accessed November 27, 2018).

10. 114th Congress. Public Law 114-255. 21st Century Cures Act. (last accessed August 30, 2018).

11. P.J. Neumann, M.S. Kamae, and J.A. Palmer, "Medicare's National Coverage Decisions for Technologies, 19992007, Health Affairs (Millwood) 27, no. 6 (2008):1620-1631.

12. Center for Medicaid and Medicare Services. Coverage with evidence development, available at <https://www.cms. gov/Medicare/Coverage/Coverage-withEvidence-Development/> (last accessed November 27, 2018); see Tunis, supra note 7 .

13. Centers for Medicare and Medicaid Services, Guidance for the Public, Industry, and CMS Staff: Coverage with Evidence Development, available at <https:// www.cms.gov/medicare-coverage-database/details/medicare-coverage-document-details.aspx? $\mathrm{MCDId}=27>$ (last accessed November 27, 2018).

14. M.W. Krucoff, R.G. Brindis, P.K. Hodgson, Mj.J. Mack, and D.R. Holmes, Jr., "Medical Device Innovation: Prospective Solutions for an Ecosystem in Crisis. Adding a Professional Society Perspective," JACC: Cardiovascular Interventions 5, no. 7 (2012):790-796; R.A. Lindor, S.J. Allocco, L. Cheatham, et al. "Regulatory and Reimbursement Innovation," Science Translational Medicine 5, no. 176 (2013):176cm3.

15. D.A. Messner and S.R. Tunis, "Current and Future State of FDA-CMS Parallel Reviews, Clinical Pharmacology $ङ$ Therapeutics 91, no. 3 (2012): 383-385.

16. J.R. Ridge and S. Statz, "Exact Sciences' Experience with the FDA and CMS Parallel Review Program," Expert Review of Molecular Diagnostics 15, no. 9 (2015):1117-1124.

17. Centers for Medicare and Medicaid Services, Medicare Program Integrity Manual - Chapter 13 Local Coverage Determinations, available at $<\mathrm{https}$ :// www.cms.gov/Regulations-and-Guidance/Guidance/Manuals/.../pim83c13. pdf $>$ (last accessed November 27, 2018).

18. Memorandum of Understanding between United States Food and Drug Administration and Centers for Medicare \& Medicaid Services In: Administration $\mathrm{FaD}$, ed.2010.

19. Pilot Program for Parallel Review of Medical Products In: Administration CfMaMSFaD, ed.2011:6280810; Services DoHaH. Medicare Program: Revised Process for Making National Coverage Determinations. In: Services CfMM, ed. Federal Register2003:55634-41.
20. Chambers, supra note 6; S. Basu and J.C. Hassenplug, "Patient Access to Medical Devices - A Comparison of U.S. and European Review Processes," New England Journal of Medicine 367, no. 6 (2012):485-488.

21. J.D. Chambers, M. Chenoweth, T. Thorat, and P.J. Neumann, "Private Payers Disagree with Medicare over Medical Device Coverage about Half the Time," Health Affairs (Millwood) 34, no. 8 (2015):1376-1382.

22. D.E. Forman, M.S. Maurer, C. Boyd, et al., "Multimorbidity in Older Adults With Cardiovascular Disease," Journal of the American College of Cardiology 71, no. 19 (2018): 2149-2161.

23. S.S. Dhruva, C.M. Mazure, J.S. Ross, R.F. Redberg, "Inclusion of Demographic-Specific Information in Studies Supporting US Food \& Drug Administration Approval of High-Risk Medical Devices," JAMA Internal Medicine 177 , no. 9 (2017):1390-1391.

24. National Academies of Sciences, Engineering, and Medicine, "Strategies for Ensuring Diversity, Inclusion, and Meaningful Participation in Clinical Trials," (Washington, DC: National Academies Press, August 24, 2016), available at <https://www.ncbi.nlm.nih. gov/pubmed/27606381> (last accessed November 27, 2018); US Food and Drug Administration, "FDA Report: Collection, Analysis, and Availability of Demographic Subgroup Data for FDAApproved Medical Products," August 2013, available at <https://www.fda. gov/downloads/RegulatoryInformation/LawsEnforcedbyFDA/SignificantAmendmentstotheFDCAct/FDASIA/UCM365544.pdf> (last accessed November 27, 2018).

25. E. Richardson, "Health Policy Brief: Aligning FDA and CMS Review," Health Affairs (2015), available at <https://www.healthaffairs.org/ do/10.1377/hpb20150827.132391/full/> (last accessed December 12, 2018); F Zannad, M.L.A. Alonso Garcia, and J.S. Borer, et al. "Role of Payers in the Development of Cardiovascular Therapeutics: Misalignment Between Approval and Reimbursement," Journal of the American College of Cardiology 70, no. 22 (2017):2822-2830

26. J. Rickard, H. Michtalik, R. Sharma, et al., "Use of Cardiac Resynchronization Therapy in the Medicare Population," AHRQ Technology Assessment, Rockville, MD, 2015.

27. D.B. Kramer and D.A. Steinhaus, "Cardiac Resynchronization Therapy in Older Patients: Age Is Just a Number, and Yet...," Journal of Cardiac Failure
22, no. 12 (2016): 978-980; M.B. Elming, J.C. Nielsen, J. Haarbo, et al., "Age and Outcomes of Primary Prevention Implantable Cardioverter-Defibrillators in Patients With Nonischemic Systolic Heart Failure," Circulation 136, no. 19 (2017):1772-1780.

28. US Food and Drug Administration, Medical Devices: Databases, 510(k) premarket notification, available at <https://www.accessdata.fda.gov/ scripts/cdrh/cfdocs/cfpmn/pmn.cfm> (last acessed November 27, 2018).

29. Institute of Medicine, Medical Devices and the Public's Health: The FDA $510(\mathrm{k})$ Clearance Process at 35 Years, Washington, DC: National Academies Press, 2011; B.M. Ardaugh, S.E. Graves, and R.F. Redberg, "The $510(\mathrm{k})$ Ancestry of a Metal-on-Metal Hip Implant," New England Journal of Medicine 368, no. 2 (2013): 97-100.

30. See Chambers, supra note 6.

31. Forman, supra note 22.

32. S.S. Dhruva and R.F. Redberg, "Variations Between Clinical Trial Participants and Medicare Beneficiaries in Evidence used for Medicare National Coverage Decisions," Archives of Internal Medicine 168, no. 2 (2008):136-140.

33. M. Tisminetzky, E.A. Bayliss, J.S. Magaziner, et al., "Research Priorities to Advance the Health and Health Care of Older Adults with Multiple Chronic Conditions," Journal of the American Geriatrics Society 65, no. 7 (2017):15491553;; A.S. Bierman and M.E. Tinetti, "Precision Medicine to Precision Care: Managing Multimorbidity, Lancet 388 , no. 10061 (issue number?)(2016): 2721-2723.

34. Department of Health and Human Services, Office of Inspector General, Local Coverage Determinations Create Inconsistency in Medicare Coverage, OEI-O111-00500, January 2014.

35. Centers for Medicare and Medicaid Services, Decision Memo for Positron Emission Tomography (FDG) for Solid Tumors (CAG-00181R4), available at $<$ https://www.cms.gov/medicare-coverage-database/details/nca-decisionmemo.aspx? NCAId $=263>$ (last acessed November 27, 2018)

36. Centers for Medicare and Medicaid Services, Decision Memo for Transcatheter Aortic Valve Replacement (CAG$00430 \mathrm{~N}$ ), available at <https://www. $\mathrm{cms}$.gov/medicare-coverage-database/ details/nca-decision-memo.aspx?NCAI $\mathrm{d}=257 \& \mathrm{ver}=4 \& \mathrm{NcaName}=$ Transcathet er+Aortic+Valve+Replacement+(TAVR) $\& b c=$ ACAAAAAAIAAA $\&>$ (last acessed November 27, 2018). 